\title{
Propiedades psicométricas del Cuestionario de Motivos de Consumo de Alcohol para Argentina
}

\section{Psychometric properties of the Drinking Motives Questionnaire for the Argentinean population}

\author{
Florencia Caneto ${ }^{\mathrm{a}}$, Marcos Cupani ${ }^{\mathrm{a}, \mathrm{b}}$ y Angelina Pilatti ${ }^{\mathrm{a}} \mathrm{b}$ \\ ${ }^{a}$ Universidad Nacional de Córdoba, Córdoba, Argentina ${ }^{b}$ Consejo Nacional de Investigaciones Científicas y Técnicas (Conicet), Argentina
}

\section{Resumen}

El presente estudio tuvo como objetivo evaluar las propiedades psicométricas -validez de estructura y consistencia internade tres modelos teóricos (Drinking Motives Questionnaire [DMQ], Drinking Motives Questionnaires-Revised [DMQ-R] y Modified Drinking Motives Questionnaires-Revised [M-DMQ-R]) desarrollados para medir los motivos de consumo de alcohol y explorar la relación entre estos y la severidad de los problemas derivados. La muestra constó de 315 jóvenes y adultos jóvenes argentinos, con edades entre 18 y 40 años, que reportaron beber alcohol en los últimos tres meses. Para evaluar los modelos teóricos, se utilizaron modelos confirmatorios de análisis factorial con estimación por máxima verosimilitud. Los resultados indicaron que los modelos DMQ y DMQ-R presentaron excelentes y adecuados niveles de ajuste, respectivamente. Sin embargo, el modelo M-DMQ-R no mostró adecuado ajuste a los datos. Respecto a la validez concurrente, los motivos de mejora y de afrontamiento, pero no los motivos sociales, se asociaron a un mayor nivel de severidad de problemas con el alcohol. Los resultados indican que el DMQ representa una herramienta válida y confiable para medir los motivos de consumo de alcohol de jóvenes y adultos jóvenes de la población general argentina.

Palabras clave: consumo de alcohol, motivos, escala, propiedades psicométricas.

\begin{abstract}
The current study evaluated the psychometric properties -structural validity and internal consistency- of three theoretical models developed to assess drinking motives (Drinking Motives Questionnaire [DMQ], Drinking Motives Questionnaires-Revised [DMQ-R] y Modified Drinking Motives Questionnaires-Revised [M-DMQ-R]) and explored the relationship between different reasons for drinking and the severity of problems arising from alcohol consumption. The sample was composed by 315 youth and young adults from Argentina, aged from 18 to 40, who reported drinking alcohol in the last three months. To evaluate the theoretical models, a confirmatory factor analysis model was employed using maximum likelihood estimation. The results indicated that DMQ and DMQ-R models exhibited excellent and adequate fit indices, respectively. However, the M-DMQ-R model did not show adequate fit to the data. Regarding concurrent validity, enhancement and coping drinking motives, but not social motives, were associated to higher degrees of severity of alcohol problems. Overall, results indicated that DMQ model is a valid and reliable tool to measure drinking motives of youth and young adults from the general Argentinean population.
\end{abstract}

Keywords: alcohol consumption, motives, scale, psychometric properties.

Este trabajo ha sido posible gracias al subsidio de la Agencia Nacional de Promoción Científica y Tecnológica (Foncyt) a nombre de Angelina Pilatti (PICT 2012-1736).

Contacto: A. Pilatti. Laboratorio de Psicología, Facultad de Psicología, Universidad Nacional de Córdoba. Enrique Barros y Enfermera Gordillo s/n, Ciudad Universitaria. Córdoba, 5000. Argentina. apilatti@psyche.unc.edu.ar

Cómo citar este artículo:

Caneto, F., Cupani, M. y Pilatti, A. (2014). Propiedades psicométricas del Cuestionario de Motivos de Consumo de Alcohol para Argentina. Revista de Psicología, 24(1), 21-32. doi: 10.5354/0719-0581.2014.32871 


\section{Introducción}

El consumo abusivo de alcohol entre los adolescentes y jóvenes es uno de los problemas más importantes en salud pública a nivel mundial (World Health Organization [WHO], 2010). Estudios realizados en diferentes países occidentales (Johnston, O’Malley, Bachman y Schulenberg, 2011; Ståhlbrandt, Leifman, Johnsson y Berglund, 2010), incluida la Argentina (Garimaldi, Caneto, Vera, Tuzinkievich y Pilatti, 2013; Pilatti, Caneto, Garimaldi, Vera y Pautassi, 2014; Pilatti, Godoy, Brussino y Pautassi, 2013a; Secretaría de Programación para la Prevención de la Drogadicción y la Lucha contra el Narcotráfico [Sedronar], 2011; Vera, Caneto, Garimaldi y Pilatti, 2013), indican que entre 30\% y 50\% de adolescentes y jóvenes exhibe consumo episódico elevado (igual o mayor a 56 gramos de alcohol en una misma ocasión en mujeres y 70 gramos de alcohol en hombres).

Los adolescentes y jóvenes con estos patrones de consumo tienen una mayor probabilidad de experimentar una gran variedad de consecuencias negativas, como fallas en el rendimiento académico, desmayos, relaciones sexuales riesgosas, agresiones sexuales y físicas, accidentes (Hingson, Edwards, Heeren y Rosenbloom, 2009), toma de decisiones desventajosas y riesgosas (Mason et al., 2011), mayor impulsividad (Goudriaan, Grekin y Sher, 2011) e inclusive desarrollo de dependencia al alcohol (Dawson, Grant, Stinson y Chou, 2004). En un estudio local (Pilatti, Read et al., 2014) se encontró que casi el $25 \%$ de una muestra de jóvenes experimentó, en los tres meses previos, al menos diez consecuencias negativas (e.g., amnesia, impulsividad y toma de riesgos exacerbada, manejo de automóviles luego de beber alcohol y desarrollo de tolerancia) por el consumo de alcohol.

Una gran diversidad de estudios ha permitido identificar una serie de factores que influyen sobre el consumo de alcohol en adolescentes y jóvenes. Efectivamente, la decisión de una persona (que puede ser consciente o no) sobre si beberá o no alcohol está determinada, entre otros, por componentes genéticos, rasgos de personalidad (e.g., impulsividad), elementos culturales y sociales (e.g., modalidades de consumo de alcohol) y factores cognitivos como las expectativas hacia el alcohol (Del Boca, Darkes, Greenbaum y Goldman, 2004; Pilatti, Brussino y Godoy, 2013) y los motivos de consumo de alcohol (Engels, Wiers, Lemmers y Overbeek, 2005; Kong y Bergman, 2010; Kuntsche, Knibbe, Gmel y Engels, 2006).

Los motivos de consumo de alcohol son definidos como necesidades internas o externas que las personas buscan satisfacer bebiendo alcohol (Cooper, 1994; Kuntsche et al., 2006). Se asume que las personas consumen alcohol con el objetivo de alcanzar un tipo particular de resultado y que a determi- nados motivos subyace una cadena particular de antecedentes y consecuencias. Kuntsche, Knibbe, Gmel y Engels (2005) indican que la decisión de beber alcohol es el resultado de una combinatoria de procesos emocionales y racionales donde la decisión depende del cambio afectivo que la persona espera alcanzar bebiendo alcohol, comparado con no beber alcohol. Estos cambios pueden relacionarse tanto con los efectos directos del alcohol sobre el organismo (e.g., reducción de la tensión) como con los efectos indirectos (e.g., ser aceptado por el grupo de pares).

Existen diferentes modelos teóricos para dar cuenta de la estructura de los motivos de consumo de alcohol (Cooper, 1994; Farber, Khavari y Douglass, 1980; Sher, Walitzer, Wood y Brent, 1991) o de los contextos de consumo de alcohol, que incluyen aspectos motivacionales del uso de alcohol (Annis y Graham, 1995; Beck, Thombs y Summons 1993; O'Hare, 1997, 2001). Uno de los modelos más ampliamente aceptados para medir los motivos de consumo de alcohol deriva del trabajo de Cooper et al. El Drinking Motives Questionnaire (DMQ, Cooper, Russell, Skinner y Windle, 1992) comprende tres factores o dimensiones: mejora, social y afrontamiento. Posteriormente, y teniendo en cuenta la fuente (interna o externa) y la valencia del reforzamiento (positivo o negativo), surge el modelo revisado de cuatro factores (Drinking Motives Questionnaire-Revised [DMQ-R], Cooper, 1994). Los cuatro factores corresponden a los motivos sociales (reforzamiento positivo, guiado por factores externos a la persona; e.g., beber alcohol para celebrar), los motivos de mejora, (reforzamiento positivo, guiado por factores internos; e.g., porque es divertido), los motivos de afrontamiento (reforzamiento negativo, guiado por factores internos; e.g., para olvidar preocupaciones) y los motivos de conformidad (reforzamiento negativo, guiado por factores externos; e.g., porque es lo que la mayoría de mis amigos hace cuando nos juntamos). Más recientemente, y en base a resultados que sugerían dividir los motivos de afrontamiento en afrontamiento de la ansiedad y afrontamiento de la depresión, se agregó un quinto factor (Modified Drinking Motives Questionnaire Revised, M-DMQ-R, Grant, Stewart, O'Connor, Blackwell y Conrod, 2007).

Esta diversidad de categorías o dimensiones de motivos de consumo de alcohol aparece en la adolescencia y la juventud (Kuntsche et al., 2006) asociada a los patrones normativos de uso de alcohol encontrados en diferentes edades (Masten, Faden, Zucker y Spear, 2009; Windle et al., 2009). Al respecto, el consumo de alcohol entre los niños es experimental (Cameron, Stritzke y Durkin, 2003; Pilatti, Godoy, Brusino y Pautassi, 2013b) para aumentar en regularidad, frecuencia y cantidad durante la adolescencia (Donovan et al., 2004; Urbán, Kökönyei y Demetrovics, 2008) y de manera más mar- 
cada durante la juventud (Masten et al., 2009). A medida que aumenta el consumo de alcohol, los adolescentes y jóvenes amplían, en especificidad y en complejidad, el repertorio de motivos que pueden conducir, o no, a la decisión de tomar alcohol (Kuntsche et al., 2006).

Durante la adolescencia prevalecen los motivos de consumo de alcohol relacionados con la facilitación social y la diversión, siendo muy baja la prevalencia de motivos asociados al afrontamiento de estados negativos (Kuntsche et al., 2005; Pilatti y Brussino, 2009). En tanto que la mayoría de los adolescentes y jóvenes bebe alcohol por motivos sociales, estos motivos se asocian a un consumo moderado de alcohol y no tienen, en general, un efecto directo sobre una mayor severidad de problemas con el alcohol (Merrill y Read, 2010). Efectivamente, parecen influir de manera indirecta, vía una mayor frecuencia y mayor cantidad de consumo de alcohol, sobre los problemas asociados al consumo (Gilson et al., 2013). Por otra parte, existe una asociación entre el beber alcohol para afrontar estados negativos intensos (e.g., ansiedad o depresión) y el consumo elevado y riesgoso de alcohol (Kuntsche et al., 2005; Neighbors, Lee, Lewis, Fossos y Larimer, 2007; Read, Wood, Kahler, Maddock y Palfai, 2003). Una asociación positiva similar se ha encontrado entre los motivos de mejora y el consumo problema (Hauck-Filho, Teixeira y Cooper, 2012; Kuntsche et al., 2005; Merrill y Read, 2010). Más allá de la diversidad de categorías, una mayor motivación general para beber alcohol se asocia a mayores niveles de consumo de alcohol. Sin embargo, la relación positiva entre el consumo de alcohol y los motivos de mejora y afrontamiento es más elevada que con los motivos sociales (Kuntsche et al., 2005).

El DMQ-R fue adaptado a diferentes idiomas como italiano (Zogmaister y Castelli, 2011), portugués (Hauck-Filho et al., 2012), húngaro (Németh, Urbán, Farkas, Kuntsche y Demetrovics, 2012) y turco (Evren, Celik, Aksoy y Cetin, 2010). Asimismo, existe una versión breve de este instrumento, la DMQ-R-SF (Kuntsche y Kuntsche, 2009), que también ha sido adaptada a diferentes idiomas como el húngaro (Németh et al., 2011) y el italiano (Mazzardis, Vieno, Kuntsche y Santinello, 2010). Mezquita et al. (2011), con datos de participantes de 17 a 81 años, adaptaron el M-DMQ-R para ser utilizado en España y evaluaron el ajuste de ese modelo y del DMQ-R. Los resultados del análisis factorial confirmatorio (AFC) indicaron que el M-DMQ-R presentaba un óptimo grado de ajuste a los datos y, además, sus subescalas permitieron predecir la frecuencia y cantidad de consumo de alcohol. Puntualmente, los motivos de mejora, sociales y de afrontamiento de la ansiedad predijeron un mayor consumo los fines de semana, mientras que los motivos sociales y de afrontamiento de la ansiedad permitieron predecir un mayor consumo en días laborables.

Esta versión en español, sin embargo, no fue evaluada en Argentina o en otro país de América Latina de habla hispana. Dentro de nuestro conocimiento, en Latinoamérica solo se evaluaron las propiedades psicométricas de la versión en portugués del DMQ-R en un estudio realizado en Brasil (Hauck-Filho et al., 2012). En Argentina se cuenta con una herramienta para valorar los contextos de consumo de alcohol, que incluye aspectos motivacionales del consumo, en adolescentes de 13 a 18 años (Pilatti y Brussino, 2009). El estudio de las motivos para el consumo de alcohol aparece, sin embargo, como una línea diferenciada de los contextos de consumo (Grant et al., 2007; Mushquash, Stewart, Comeau y McGrath, 2008). Sumado a esto, y como ya se mencionó anteriormente, entre los jóvenes no solo aumenta la frecuencia y cantidad de alcohol consumida sino que también se diversifican los motivos de consumo de alcohol.

En este marco, el presente trabajo tiene como finalidad evaluar, en una muestra de jóvenes y adultos jóvenes, provenientes de la población general argentina, las propiedades psicométricas de la versión en español del M-DMQ-R adaptado al contexto argentino. Asimismo, y teniendo en cuenta la existencia de diferentes modelos teóricos, se propone evaluar también el ajuste del DMQ (Cooper et al., 1992) y del DMQ-R (Cooper, 1994) con la finalidad de examinar cuál de estos modelos presenta mejor ajuste al contexto argentino. Mezquita et al. (2011) indicaron como una limitación de su estudio no haber examinado la asociación entre los motivos de consumo con una medida de severidad de problemas con el alcohol. En este contexto, y para obtener evidencia de validez concurrente, se propone evaluar la asociación entre las diferentes dimensiones de los motivos de consumo de alcohol con la severidad de problemas con el alcohol.

\section{Método}

\section{Participantes}

Para determinar el tamaño de la muestra, se siguió el criterio de contar con un mínimo de 200 casos (Shah y Goldstein, 2006). Considerando este criterio, se invitó a participar en el estudio a cualquier persona, con edades entre 18 a 40 años, que haya bebido alcohol en los últimos tres meses y que resida en cualquier provincia de Argentina. Once casos fueron descartados por no haber completado alguno de los instrumentos y dos casos se descartaron por información confusa en las respuestas sobre consumo de alcohol (e.g., indicaron no haber bebido alcohol en el último año y luego reportaron beber alcohol una vez por semana). Así, la muestra final que- 
dó conformada por 315 participantes ( $74 \%$ mujeres, con una media de edad $=23.93$ años, $D E=5.09)$.

\section{Instrumentos}

Alcohol Use Disorders Identification Test (AUDIT). Se utilizó la versión en español del AUDIT (Morales-García et al., 2002) para detectar problemas relacionados con el consumo de alcohol. El AUDIT es uno de los instrumentos más utilizados para detectar problemas relacionados con el abuso de alcohol en adultos (Reinert y Allen, 2007) y jóvenes (Devos-Comby y Lange, 2008). Este instrumento consta de 10 ítems con una escala ordinal de respuesta donde cada ítem puede recibir una puntuación entre 0 y 4 . El puntaje total obtenido por cada participante se utilizó como variable dependiente en los análisis de regresión múltiple. Para describir a la muestra en función de la presencia, o no, de consumo problema se empleó una puntuación igual o mayor a 8 como punto de corte (Loxton, Nguyen, Casey y Dawe, 2008).

Modified Drinking Motives Questionnaire Revised (M-DMQ-R). Se utilizó la versión en español de Mezquita et al. (2011) con algunas modificaciones para adaptarla al contexto argentino. En primer lugar, la versión en español fue sometida a discusión entre los miembros del equipo de trabajo hasta obtener una versión consensuada de cada uno de los ítems. En este paso se tuvieron en cuenta las diferencias lingüísticas y culturales entre la población de origen (España) y la población destino (Argentina). Luego, se realizaron entrevistas focales individuales a seis sujetos con el propósito de evaluar los ítems del instrumento y determinar cualquier dificultad en la comprensión de los ítems. En base a los resultados de estas entrevistas se realizaron los ajustes de redacción necesarios. Para responder, los participantes debían indicar, en una escala Likert de cinco puntos (desde $1=$ casi nunca o nunca hasta 5 = casi siempre o siempre), la frecuencia con que consumían alcohol por el motivo especificado en cada ítem. Mezquita et al. (2011) reportaron los siguientes valores de consistencia interna: social $\alpha=.78$; afrontamiento de la depresión $\alpha=.88$; mejora $\alpha=.82$; conformidad $\alpha=.75$ $\mathrm{y}$ afrontamiento de la ansiedad $\alpha=.63$.

\section{Procedimientos}

Los datos se obtuvieron mediante la aplicación de una encuesta computarizada desarrollada en un sitio web seguro del Servidor de Encuestas de la Universidad Nacional de Córdoba (Argentina). El procedimiento de encuesta en línea informa a los participantes si dejaron una pregunta sin responder, previniendo el envío de encuestas con datos incompletos. La invitación a participar del estudio contenía un enlace para acceder a la encuesta en línea que incluía el formulario de consentimiento informado. Completar la encuesta tomaba, aproximadamente, 30 a 40 minutos. Previo a la difusión de la encuesta, se realizó un piloto con 10 jóvenes, que no formaron parte de la muestra final, para obtener información acerca de la claridad de los ítems de los diferentes cuestionarios y del tiempo necesario para completar la encuesta.

\section{Análisis de datos}

En primer lugar, a partir de frecuencias y porcentajes, se realizó una exploración del comportamiento de las variables para describir la severidad de los problemas asociados al consumo de alcohol (AUDIT).

Luego, se efectuaron AFC con el objetivo de evaluar la estructura interna del cuestionario de motivos de consumo de alcohol. Se examinó y comparó el ajuste de tres modelos: (i) el Modelo DMQ, compuesto por tres factores latentes correlacionados (social, mejora y afrontamiento) y 15 ítems como variables observables con sus respectivos errores de medición; (ii) el Modelo DMQ-R, compuesto por cuatro factores correlacionados (social, mejora, afrontamiento y conformidad) y 20 ítems como variables observables con sus respectivos errores de medición; y (iii) el Modelo M-DMQ-R, conformado por cinco factores correlacionados (social, mejora, conformidad, afrontamiento ansiedad y afrontamiento de depresión) y 28 ítems como indicadores observables con sus respectivos errores de medición.

Para este análisis se utilizó el software EQS 6.1 y el método de estimación empleado fue el de máxima verosimilitud (ML) con la corrección robusta de Satorra-Bentler (S-B; Bentler, 2006; Satorra, 2002). Este método de estimación es el más adecuado cuando, como en el presente estudio, los datos observados se encuentran en escala ordinal y no se encuentra normalidad multivariada (Bentler, 2006; Mezquita et al., 2011).

Para evaluar el ajuste de los modelos se emplearon múltiples indicadores. Específicamente, se utilizó el estadístico $\chi^{2}$, el índice de ajuste comparativo (CFI) y el error cuadrático medio de aproximación (RMSEA). Los valores utilizados para la bondad de ajuste del modelo fueron los siguientes: índice CFI, valores entre .90 y .95 o superiores son considerados como ajustes aceptables a excelentes para el modelo; y RMSEA, valores entre .05 y .08 (Hu y Bentler, 1999). Por último, se tuvieron en cuenta los coeficientes estandarizados $(\beta)$ de cada variable observada en la variable latente (Hair, Black, Babin, Anderson y Tatham, 2006). 
Luego se calcularon los coeficientes Alfa de Cronbach para examinar la consistencia interna de cada subescala. Finalmente, se realizó una serie de análisis de regresión por pasos para examinar la validez concurrente del instrumento. Específicamente, se evaluó el poder predictivo de las subescalas de motivos de consumo (variables predictores) sobre la severidad de los problemas con el alcohol (medido con el AUDIT, variable criterio). En cada caso, se controló el efecto del sexo (hombres $=1$, mujeres $=2$ ) y la edad.

\section{Resultados}

\section{Análisis descriptivos}

Severidad de problemas con el alcohol (AUDIT). La muestra obtuvo una puntuación media en el AUDIT de 5.43 puntos $(D E=3.98)$; el $25.4 \%$ de los participantes obtuvo puntuaciones que indican consumo problemático (igual o mayor a 8), mientras que el resto obtuvo puntuaciones que reflejan modalidades no problemáticas de consumo de alcohol (igual o menor a 7).

\section{Análisis factorial confirmatorio}

Se examinaron todos los ítems con el fin de evaluar la calidad de la base de datos. Para comprobar los supuestos de normalidad de la muestra se realizaron análisis de asimetría y curtosis para cada ítem. Diecinueve ítems presentaron índices de curtosis y asimetría mayor a +2.00 y -2.00 , lo cual es considerado por la literatura como inadecuados (George y Mallery, 2011). La tabla 1 presenta los estadísticos descriptivos de los ítems (media, desviación típica, curtosis y asimetría). Estos resultados, al igual que los observados por Mezquita et al. (2011), indican que los ítems de motivos de consumo no presentan una distribución normal. Un último análisis consistió en el diagnóstico de multicolinealidad entre los ítems a los fines de estimar la existencia de variables altamente correlacionadas o redundantes (correlaciones inter-ítem de .80 o superiores). Los resultados observados indican una correlación de .79 entre el ítem para encajar en un grupo que me gusta y para no sentirme excluido.

La tabla 2 muestra las cargas factoriales estandarizadas de cada uno de los ítems agrupados según cada uno de los tres modelos analizados (DMQ, DMQ-R y M-DMQ-R).

Modelo DMQ: tres factores (social, mejora y afrontamiento). Los estadísticos de ajuste indican un aceptable grado de ajuste a los datos: Satorra-Bentler $\chi_{(87)}^{2}=265.33$, $p<.001 ; \mathrm{CFI}=.912$; RMSEA $=.08$, IC 90\% [.07, .09]. Las correlaciones observadas entre los tres factores fueron las siguientes: el factor social correlacionó positiva y significati- vamente con los factores mejora $(r=.60, p \leq .001)$ y afrontamiento $(r=.47, p \leq .001)$, mientras que los factores mejora y afrontamiento también presentaron una correlación positiva y significativa $(r=.68, p \leq .001)$. Las cargas factoriales estandarizadas $(p \leq .05)$ en el factor social oscilaron entre $.34 \mathrm{y}$ .88 , en el factor mejora entre .68 y .85 , y en el factor afrontamiento entre .42 y .82. Al eliminar el ítem como una manera de celebrar del factor social, que presentó una carga factorial pequeña y explicó solo un $11 \%\left(\mathrm{R}^{2}\right)$, se observó una mejora en el grado de ajuste (Satorra-Bentler $\chi_{(87)}^{2}=148.15, p<.001$; $\mathrm{CFI}=.964 ;$ RMSEA $=.05$, IC 90\% $[.03, .06]$.

Modelo DMQ-R: cuatro factores (social, mejora, afrontamiento y conformidad). Los valores del grado de ajuste estuvieron ligeramente debajo de los valores recomendados: Satorra-Bentler $\chi_{(164)}^{2}=481.94, p<.001$; CFI $=.886$; RMSEA $=.08$, IC $90 \%[.073, .09]$. El factor social correlacionó positiva y significativamente con los factores mejora $(r=.60$, $p \leq .001)$, afrontamiento $(r=.47, p \leq .001)$ y conformidad $(r=.46, p \leq .001)$. El factor mejora presentó una correlación positiva y significativa con los factores afrontamiento $(r=$ $.68, p \leq .001)$ y conformidad $(r=.36, p \leq .001)$. Los factores afrontamiento y conformidad también presentaron una correlación positiva y significativa $(r=.49, p \leq .001)$. Las cargas factoriales estandarizadas $(p \leq .05)$ en el factor social oscilaron entre .29 y .78 , en el factor mejora entre .68 y .86 , en el factor afrontamiento entre .41 y $.86 \mathrm{y}$ en el factor conformidad entre 61 y .97. Un examen de los índices de modificación indicó que un cambio en los parámetros estimados entre los errores pertenecientes al par de ítems para encajar en un grupo que me gusta y para no sentirme excluido, del factor conformidad, permitía un mejor grado de ajuste de los datos. Considerando que un análisis previo indicó que estos dos ítems presentaban multicolinealidad $(r=.79)$, se decidió eliminar el ítem para no sentirme excluido del modelo. Por otro lado, y como se observó en el análisis anterior, el ítem como una manera de celebrar, del factor social, presentó una carga factorial pequeña y explicó sólo un $8 \%\left(R^{2}\right)$. La eliminación de estos dos ítems se tradujo en una mejora del grado de ajuste: Satorra-Bentler $\chi_{(129)}^{2}=311.56, p<.001 ; \mathrm{CFI}=.908$; RMSEA $=.07$, IC $90 \%[.06, .08]$.

Modelo M-DMQ-R: cinco factores (social, mejora, afrontamiento de la ansiedad, afrontamiento de la depresión y conformidad). Los resultados indicaron que el modelo no presentaba un adecuado grado de ajuste a los datos: Satorra-Bentler $\chi_{(340)}^{2}=651.7, p<.001$; CFI $=.745$; RMSEA $=.05$, IC $90 \%[.05, .06]$. El factor social correlacionó positiva y significativamente con los factores mejora $(r=.60, p \leq$ $.001)$, afrontamiento de la ansiedad $(r=.47, p \leq .001)$, afrontamiento de la depresión $(r=.33, p \leq .001)$ y conformidad $(r=.46, p \leq .001)$. El factor mejora presentó una correlación 
Tabla 1

Estadísticos descriptivos de los ítems (media, desviación típica, curtosis y asimetría)

\begin{tabular}{|c|c|c|c|c|}
\hline & Media & DT & Asimetría & Curtosis \\
\hline Como una manera de festejar o celebrar. & 3,70 & 1.095 & -0.558 & -0.651 \\
\hline Para relajarme. & 1.79 & 0.987 & 1.369 & 1.463 \\
\hline Porque me gusta la sensación que me genera. & 2.04 & 1.200 & 1.010 & -0.030 \\
\hline Porque es lo que la mayoría de mis amigos hacen cuando nos juntamos & 2.06 & 1.254 & 0.978 & -0.222 \\
\hline Para olvidar mis preocupaciones. & 1.44 & 0.840 & 2.314 & 5.345 \\
\hline Porque es excitante. & 1.58 & 1.007 & 1.867 & 2.734 \\
\hline Para ser sociable & 1.76 & 1.079 & 1.502 & 1.528 \\
\hline Porque me hace sentir más confiado o más seguro de mí mismo. & 1.63 & 1.049 & 1.778 & 2.392 \\
\hline Para sentirme intoxicado o borracho & 1.30 & 0.659 & 2.879 & 10.295 \\
\hline Porque es algo que se acostumbra hacer en ocasiones especiales & 2.35 & 1.325 & 0.618 & -0.884 \\
\hline Porque me ayuda cuando me siento nervioso. & 1.33 & 0.781 & 2.792 & 7.890 \\
\hline Porque es divertido. & 2.27 & 1.254 & 0.646 & -0.774 \\
\hline Porque hace que las reuniones sociales sean más entretenidas & 2.46 & 1.345 & 0.452 & -1.128 \\
\hline Para sentirme mejor cuando estoy de mal humor & 1.42 & 0.835 & 2.426 & 6.157 \\
\hline Para gustar a los demás. & 1.22 & 0.595 & 3.352 & 12.457 \\
\hline Para calmar mi dolor & 1.21 & 0.553 & 3.340 & 13.443 \\
\hline Porque me ayuda cuando me siento deprimido. & 1.30 & 0.608 & 2.654 & 9.582 \\
\hline Para que los otros no me carguen o no se burlen de mi si no tomo. & 1.06 & 0.276 & 4.992 & 26.561 \\
\hline Para reducir mi ansiedad & 1.30 & 0.671 & 3.320 & 13.814 \\
\hline Para parar de pensar de forma obsesiva en ciertas cosas. & 1.27 & 0.664 & 3.042 & 10.358 \\
\hline Para dejar de pensar negativamente sobre mí mismo & 1.20 & 0.626 & 3.744 & 15.086 \\
\hline Para ayudar a sentirme más positivo/a & 1.18 & 0.576 & 4.012 & 18.146 \\
\hline Para dejar de sentirme desesperanzado/a sobre el futuro & 1.12 & 0.449 & 4.749 & 27.333 \\
\hline Porque mis amigos me presionan para que tome & 1.08 & 0.339 & 5.705 & 38.929 \\
\hline Para encajar en un grupo que me gusta. & 1.13 & 0.426 & 3.973 & 17.895 \\
\hline Porque me hace sentir bien. & 1.85 & 1.063 & 1.269 & 0.884 \\
\hline Para olvidar recuerdos dolorosos. & 1.23 & 0.546 & 3.274 & 14.965 \\
\hline Para no sentirme excluido. & 1.16 & 0.490 & 4.105 & 20.750 \\
\hline
\end{tabular}

positiva y significativa con los factores afrontamiento de la ansiedad $(r=.64, p \leq .001)$, afrontamiento de la depresión $(r=.54, p \leq .001)$ y conformidad $(r=.36, p \leq$ $.001)$. El factor conformidad estuvo positiva y significativamente correlacionado con los factores afrontamiento de la ansiedad $(r=.51, p \leq .001)$ y afrontamiento de la depresión $(r=.43, p \leq .001)$. Finalmente, el factor afrontamiento de la ansiedad correlacionó con el factor afrontamiento de la depresión $(r=.67, p \leq .001)$. Las cargas factoriales estandarizadas $(p \leq .05)$ en el factor social oscilaron entre .30 y.76, en el factor mejora entre .54 y .80 , en el factor afrontamiento ansiedad entre .31 y .76, en el factor afrontamiento depresión entre .61 y .82 y en el factor conformidad, entre .37 y .90 . Un examen de los índices de modificación indicó que un cambio en los parámetros estimados entre los errores pertenecientes al ítem para gustar a los demás, de la escala conformidad, y los ítems para ser sociable, porque es excitante, para olvidar mis preocupaciones, para sentirme mejor cuando estoy de mal humor, para calmar mi dolor, para dejar de pensar negativamente sobre mí mismo y porque me hace sentir más confiado o más seguro de mí mismo, mejoraría el ajuste a los datos. Se eliminó del modelo el ítem para gustar a los demás, sin embargo, no se observó una mejoría en el grado de ajuste: Satorra-Bentler $\chi_{(340)}^{2}=651.7, p<.001$; CFI $=$ .745 ; RMSEA $=.05$, IC 90\% $[.05, .06]$. 
Tabla 2

Cargas factoriales estandarizadas de cada uno de los ítems, agrupados según cada uno de los tres modelos analizados (DMQ, $D M Q-R$ y $M-D M Q-R)$

\begin{tabular}{|c|c|c|c|}
\hline & $\begin{array}{c}\beta \\
\text { DMQ }\end{array}$ & $\begin{array}{c}\beta \\
\text { DMQ-R }\end{array}$ & $\begin{array}{c}\beta \\
M-D M Q-R\end{array}$ \\
\hline \multicolumn{4}{|l|}{ SOCIAL } \\
\hline Como una manera de festejar o celebrar & .34 & .29 & .30 \\
\hline Porque es lo que la mayoría de mis amigos hace cuando nos juntamos & .51 & .52 & .484 \\
\hline Para ser sociable & .63 & .74 & .697 \\
\hline Porque es algo que se acostumbra hacer en ocasiones especiales & .47 & .43 & .399 \\
\hline Porque hace que las reuniones sociales sean más entretenidas & .88 & .78 & .741 \\
\hline \multicolumn{4}{|l|}{ MEJORA } \\
\hline Porque me gusta la sensación que me genera & .75 & .75 & .683 \\
\hline Porque es excitante & .76 & .75 & .664 \\
\hline Para sentirme intoxicado o borracho & .68 & .68 & .537 \\
\hline Porque es divertido & .85 & .86 & .799 \\
\hline Porque me hace sentir bien & .75 & .74 & .701 \\
\hline \multicolumn{4}{|l|}{ CONFORMIDAD } \\
\hline Para gustar a los demás & & .79 & .781 \\
\hline Para que los otros no me carguen o no se burlen de mí si no tomo & & .77 & .440 \\
\hline Porque mis amigos me presionan para que tome & & .61 & .318 \\
\hline Para encajar en un grupo que me gusta & & .95 & .682 \\
\hline Para no sentirme excluido & & .97 & .90 \\
\hline \multicolumn{4}{|l|}{ AFRONTAMIENTO } \\
\hline \multicolumn{4}{|l|}{ AFRONTAMIENTO DE LA ANSIEDAD } \\
\hline Para relajarme & .43 & .41 & .297 \\
\hline Porque me hace sentir más confiado o más seguro de mí mismo & .77 & .78 & .688 \\
\hline Porque me ayuda cuando me siento nervioso & .82 & .86 & .743 \\
\hline Para reducir mi ansiedad & & & .631 \\
\hline \multicolumn{4}{|l|}{ AFRONTAMIENTO DE LA DEPRESIÓN } \\
\hline Para olvidar mis preocupaciones & .77 & .74 & .716 \\
\hline Para sentirme mejor cuando estoy de mal humor & .81 & .77 & .652 \\
\hline Para calmar mi dolor & & & .611 \\
\hline Porque me ayuda cuando me siento deprimido & & & .709 \\
\hline Para parar de pensar de forma obsesiva en ciertas cosas & & & .767 \\
\hline Para dejar de pensar negativamente sobre mí mismo & & & .823 \\
\hline Para ayudar a sentirme más positivo/a sobre cosas que suceden en mi vida & & & .773 \\
\hline Para dejar de sentirme desesperanzado/a sobre el futuro & & & .718 \\
\hline Para olvidar recuerdos dolorosos & & & .619 \\
\hline
\end{tabular}

\section{Análisis de consistencia interna}

Se calculó el coeficiente Alfa de Cronbach para examinar la consistencia interna de cada una de las subescalas que componen cada uno de los tres modelos. Las subescalas del MDMQ-R presentaron los siguientes valores de consistencia interna: afrontamiento de la ansiedad $\alpha=.66$; social $\alpha=.71$; mejora $\alpha=.81$; conformidad $\alpha=.77$; $y$ afrontamiento de la depresión $\alpha=.90$. La subescala afrontamiento (que agrupa afrontamiento de la ansiedad y depresión), correspondiente a los modelos DMQ y DMQ-R, presentó una consistencia interna de $\alpha=.76$. Luego, se calculó la consistencia interna de las subescalas social y conformidad, eliminando los ítems que mostraron un desempeño irregular durante el AFC.
Puntualmente, de la escala social se eliminó el ítem como una manera de celebrar (resultados AFC modelos DMQ y DMQ-R) y de la escala conformidad se eliminó el ítem para no sentirme excluido (resultados AFC modelo DMQ-R). Mientras que la confiabilidad de la escala social no presentó modificaciones, se observó una reducción importante en la consistencia interna de la escala conformidad (de .77 a .66).

\section{Análisis de regresión: validez concurrente}

Para llevar adelante el análisis de regresión por pasos; se ingresaron en el primer paso las variables sociodemográficas y en el segundo paso cada una de las escalas de los dos modelos que mostraron mejor grado de ajuste en el AFC (modelos 
DMQ y DMQ-R). La variable criterio fue el nivel de severidad de problemas con el alcohol medido con el AUDIT. Estos resultados se presentan en la tabla 3.

Modelo DMQ. En el primer paso, la edad y el sexo de los participantes explicaron un .09\% de la varianza de la variable criterio, $F$ cambio $(2,312)=14.91, p<.001$. Los participantes más jóvenes $(\beta=-.23, t=4.14, p<.001)$ y los de sexo masculino $(\beta=-.17, t=3.04, p<.001)$ presentaron mayor severidad de problemas asociados al uso de alcohol. En el segundo paso, el monto de varianza explicada al ingresar las tres subescalas del DMQ, aumentó al 25\%, $F$ cambio (3, $309)=22.58, p<.001$. La mayor frecuencia de consumo de alcohol por motivos de mejora $(\beta=.26, t=3.53, p<.001) \mathrm{y}$ por motivos de afrontamiento $(\beta=.17, t=2.52, p<.05)$ se asociaron a un mayor nivel de severidad en los problemas con el alcohol. Se registró, además, una disminución en el coeficiente de regresión de la edad (de -.23 a -.15), indicando la mediación parcial de los motivos sobre el efecto de la edad sobre la variable criterio.

Modelo DMQ-R. En el primer paso, las variables sociodemográficas explicaron un .09\% de la varianza de la variable criterio, $F$ cambio $(2,312)=14.91, p<.001$. Los participantes más jóvenes $(\beta=-.23, t=4.14, \mathrm{p}<.001)$ y los de sexo masculino $(\beta=-.17, t=3.04, p<.001)$ presentaron mayor severidad de problemas con el alcohol. En el segundo paso, el ingreso de las cuatro subescalas del DMQ-R se tradujo en un incremento de la varianza explicada del 19\%, $F$ cambio $(4,308)=19.83, p<.001$. La mayor frecuencia de consumo de alcohol en respuesta a de mejora $(\beta=.24, t=3.15, p<.01)$ $\mathrm{y}$ de afrontamiento $(\beta=.25, t=3.49, p<.001)$ se asociaron a un mayor nivel de severidad en los problemas con el alcohol. Por otro lado, un mayor nivel de motivos de conformidad se asoció de manera negativa con el nivel de severidad $(\beta=-.19$, $t=3.11, p<.01)$. Además, se observó una disminución en el coeficiente de regresión de la edad (de -.23 a -.15), indicando la mediación parcial de los motivos sobre el efecto de la edad en la variable criterio.

\section{Discusión}

El presente estudio tuvo como finalidad examinar las propiedades psicométricas de la versión en español del M-DMQ-R (Mezquita et al., 2011) adaptada al contexto argentino. Para esto se utilizaron datos de una muestra de jóvenes y adultos de la población general argentina. Asimismo, se buscó comparar este modelo con el DMQ-R y con el DMQ para determinar cuál presentaba mejor adecuación a nuestro medio. Para evaluar el ajuste de los tres modelos teóricos propuestos se realizaron tres análisis factoriales confirmatorios. Los datos presentaron, al igual que en estudios previos (Mezquita et al., 2011), una distribución no normal, por lo que se estimó el ajuste de los modelos utilizando el método adecuado a esta característica de los datos.

Los resultados del análisis factorial confirmatorio indicaron que el modelo de tres factores, correspondiente al DMQ (Cooper, 1992), fue el que presentó mejor grado de ajuste a los datos. Tras la eliminación de un único ítem con baja carga factorial, se observó una mejora del grado de ajuste desde valores adecuados a valores excelentes, sin que se altere la consistencia interna de la escala. En un estudio previo, donde los mismos ítems se utilizaron para medir los motivos de realización de juegos de apuestas, también se observó un funcionamiento irregular con este ítem (Dechant, 2013).

En un primer momento, y de manera similar a lo observado en la adaptación al portugués (Hauck-Filho et al., 2012), el DMQ-R (modelo de cuatro factores) no presentó un adecuado grado de ajuste a los datos. Al eliminar dos ítems del modelo, uno por baja carga factorial y otro por presentar multicolinealidad, mejoró el grado de ajuste a los datos. Sin

Tabla 3

Análisis de regresión múltiple para predecir severidad de problemas con el alcohol

\begin{tabular}{|c|c|c|c|c|c|}
\hline & & \multicolumn{2}{|c|}{ DMQ } & \multicolumn{2}{|c|}{ DMQ-R } \\
\hline & & $\beta$ & $t$ & $\beta$ & $t$ \\
\hline \multirow[t]{2}{*}{$1^{\circ}$} & Sexo & -.166 & $3.039^{* *}$ & -.166 & $3.039^{* *}$ \\
\hline & Edad & -.226 & $4.144^{* * *}$ & -.226 & $4.144^{* * *}$ \\
\hline \multirow[t]{6}{*}{$2^{\circ}$} & Sexo & -.189 & $3.798^{* * *}$ & -.175 & $3.545^{* * *}$ \\
\hline & Edad & -.147 & $2.891^{* *}$ & -.145 & $2.898^{* *}$ \\
\hline & Mejora & .264 & $3.526^{* * *}$ & .235 & $3.150^{* *}$ \\
\hline & Social & .022 & 0.340 & .089 & 1.341 \\
\hline & Afrontamiento & .170 & $2.522^{*}$ & .248 & $3.494^{* * *}$ \\
\hline & Conformidad & - & - & -.185 & $3.111^{* *}$ \\
\hline
\end{tabular}

Nota: ${ }^{*} p \leq .05,{ }^{* *} p \leq .005 \mathrm{y}{ }^{* * *} p \leq .001$. 
embargo, se observó una baja considerable en la consistencia interna de la escala conformidad luego de eliminar el ítem para no sentirme excluido.

Finalmente, el modelo de cinco factores, a diferencia de lo observado en la versión original (Grant et al., 2007) y en la adaptación al español (Mezquita et al., 2011), no logró niveles óptimos de ajuste a las puntuaciones de los jóvenes y adultos participantes. Respecto a la confiabilidad, cuatro de las cinco subescalas del M-DMQ-R presentaron valores de consistencia interna superiores a .70, sin embargo, la escala afrontamiento de la ansiedad presentó un valor bajo de confiabilidad. Mezquita et al. (2011) también encontraron valores más bajos de confiabilidad (.63) en esta subescala. Interesantemente, y de manera similar a lo encontrado en la adaptación al portugués del DMQ-R (Hauck-Filho et al., 2012), se encontraron mejores índices de confiabilidad en la escala que mide los motivos de afrontamiento en una única dimensión (DMQ y DMQ-R). Estos resultados sugieren que la consideración conjunta de los motivos de afrontamiento ofrece una medición más confiable de esta dimensión. Respecto a los valores de confiabilidad hallados en este estudio, vale mencionar que hay diferentes criterios respecto a los valores adecuados de consistencia interna. Hogan (2004) sugiere que valores superiores a .70 pueden ser considerados como aceptables si la escala se utiliza con fines de investigación, pero insuficiente si se pretende emplear en el ámbito profesional, donde son deseables valores de .90 o superiores.

En función de los resultados del análisis factorial confirmatorio, se analizó la validez concurrente de los modelos que mostraron un óptimo grado de ajuste a los datos (DMQ y DMQ-R) respecto a los problemas con el uso de alcohol. Estos resultados indicaron que los motivos de mejora y de afrontamiento, pero no los motivos sociales, fueron los mejores predictores de la severidad de problemas con el alcohol (mayor puntuación en el AUDIT). Estos resultados coinciden, en parte, con resultados previos. Específicamente, Grant et al. (2007) encontraron que los motivos de mejora son los mejores predictores de la frecuencia y cantidad de consumo de alcohol, mientras que Mezquita et al. (2011) encontraron que los motivos de mejora son el mejor predictor de la cantidad de consumo durante el fin de semana. Contrariamente, en la adaptación al portugués, encontraron que todos los motivos, incluidos los sociales, predecían de manera similar la frecuencia y cantidad de consumo de alcohol (Hauck-Filho et al., 2012). Llamativamente, y al igual que en los estudios previos, se encontró que los motivos de conformidad presentaron un efecto negativo sobre los indicadores de consumo de alcohol (Grant et al., 2007; Hauck-Filho et al., 2012; Mezquita et al., 2011). En todos los casos, y considerando la nula o baja correlación bivariada entre esta escala y los indicadores de consumo, este efecto negativo a nivel multivariado puede deberse a un efecto de supresión.

En conjunto, los resultados del presente trabajo sugieren que el modelo teórico propuesto en el DMQ (tres factores) es el que presenta mejor grado de ajuste a los datos de esta muestra de jóvenes y adultos bebedores provenientes de la población general argentina. Efectivamente, este modelo presentó un excelente grado de ajuste a los datos y valores de consistencia interna entre aceptables y adecuados para sus tres escalas. Sumado a esto, dos de las tres escalas están asociadas a una mayor severidad de problemas con el alcohol, reflejando la capacidad de este instrumento para discriminar entre participantes con diferentes niveles de problemas con el alcohol.

Otro resultado interesante es que la cuarta parte de los participantes presentó puntuaciones en el AUDIT que reflejan modalidades problemáticas de consumo de alcohol. Estos resultados destacan la elevada prevalencia de consumo elevado de alcohol y, relacionado con esto, el gran porcentaje de jóvenes y adultos jóvenes que presenta puntuaciones que indican problemas con el consumo de alcohol.

Los resultados de este trabajo deben ser interpretados en el marco de algunas limitaciones. Aunque se procuró formar una muestra con participantes de la población general argentina, la muestra estuvo conformada por un mayor porcentaje de mujeres que de varones, y es posible que los resultados se encuentren sesgados. Futuros estudios deberían contemplar realizar los análisis aquí propuestos con una muestra más equilibrada en relación al sexo de los participantes. Asimismo, por el tipo de preguntas incluidas en la encuesta, no fue posible distinguir entre residentes en territorio argentino que hayan nacido en Argentina y aquellos que nacieron en otros países. Sumado a esto, y aunque la muestra presenta un tamaño aceptable de acuerdo al criterio de 200 casos (Shah y Goldstein, 2006), otros criterios establecen que un tamaño aceptable es aquel que cuenta con 10 casos por parámetro a estimar (Kline, 2011). En este trabajo se cumple este criterio para el modelo DMQ (tres factores), pero no para los modelos restantes. En este contexto se sugiere la realización de nuevos estudios con una muestra de mayor tamaño que posibilite, además, examinar la invarianza factorial a través de diferentes grupos de edad y también entre varones y mujeres. Por otro lado, los motivos de consumo y los problemas asociados al consumo fueron medidos en la misma medición. De esta manera, se limita la posibilidad de realmente predecir la severidad de problemas asociados al consumo de alcohol en función del tipo de motivos. Se sugiere realizar un nuevo estudio prospectivo donde los motivos sean medidos con anterioridad a los problemas para, efectivamente, establecer la capacidad predictiva de los motivos de consumo 
para el diagnóstico de problemas con el alcohol. Se destaca, sin embargo, el esfuerzo realizado para obtener información de la población general argentina y no exclusivamente de población universitaria como usualmente se utiliza en este tipo de estudios.

Más allá de estas limitaciones, y sin descuidar los aspectos referidos a la representatividad de la muestra y al diseño transversal empleado, los resultados aquí reportados son interesantes porque aportan evidencia acerca de las adecuadas propiedades psicométricas del DMQ y, en menor medida, también del DMQ-R. Puntualmente, la confirmación de la estructura interna de este instrumento posibilita el uso de esta herramienta en el contexto argentino, especialmente en el marco de investigaciones centradas en la detección de casos en riesgo para el desarrollo de patrones de abuso de alcohol.

\section{Referencias}

Annis, H. M. y Graham, J. M. (1995). Profile types on the Inventory of Drinking Situations: Implications for relapse prevention counseling. Psychology of Addictive Behaviors, 9(3), 176. doi: 10.1037//0893-164X.9.3.176

Beck, K. H., Thombs, D. L. y Summons, T. G. (1993). The social context of drinking scale: Construct validation and relationship to indicants of abuse in adolescent population. Addictive Behaviors, 18, 159-169.

Bentler, P. (2006). EQS 6 structural equations program manual. Encino, CA: Multivariate Software, Inc.

Cameron, C. A., Stritzke, W. G. y Durkin, K. (2003). Alcohol expectancies in late childhood: An ambivalence perspective on transitions toward alcohol use. Journal of Child Psychology and Psychiatry, 44(5), 687-698. doi: 10.1111/1469-7610.00155

Cooper, M. L. (1994). Motivations for alcohol use among adolescents: Development and validation of a Four-Factor Model. Psychological Assessment, 6(2), 117-128. doi: 10.1037/1040-3590.6.2.117

Cooper, M. L., Russell, M., Skinner, J. B. y Windle, M. (1992). Development and validation of a three-dimensional measure of drinking motives. Psychological Assessment, 4(2), 123-132. doi: $10.1037 / 1040-3590.4 .2 .123$

Dawson, D. A., Grant, B. F., Stinson, F. S. y Chou, P. S. (2004). Another look at heavy episodic drinking and alcohol use disorders among college and noncollege youth. Journal of Studies on Alcohol 65, 477-488.

Dechant, K. (2013). Show me the money: Incorporating financial motives into the Gambling Motives Questionnaire. Journal of Gambling Studies, june, 1-17. doi: 10.1007/s10899-013-9386-5

Del Boca, F. K., Darkes, J., Greenbaum, P. E. y Goldman, M. S. (2004). Up close and personal: Temporal variability in the drinking of individual college students during their first year. Journal of consulting and clinical psychology, 72(2), 155-164.

Devos-Comby, L. y Lange, J. E. (2008). Standardized measures of alcohol-related problems: A review of their use among college students. Psychology of Addictive Behaviors, 22(3), 349-361. doi: 10.1037/0893-164X.22.3.349

Donovan, J. E., Leech, S. L., Zucker, R. A., Loveland-Cherry, C. J., Jester, J. M., Fitzgerald, H. E., ... y Looman, W. S. (2004). Really underage drinkers: Alcohol use among elementary students. Alcoholism: Clinical and Experimental Research, 28, 341-349. doi:10.1097/01.ALC.0000113922.77569.4E

Engels, R. C. M. E., Wiers, R. W., Lemmers, L. y Overbeek, G. (2005). Drinking motives, alcohol expectancies, self-efficacy, and drinking patterns. Journal on Drug Education, 35, 147166. doi: 10.2190/6Q6B-3LMA-VMVA-L312

Evren, C., Celik, S., Aksoy, R. y Cetin, T. (2010). Factorial structure, reliability and validity of the turkish version of the Drinking Motives Questionnaire-Revised in Male Alcohol Dependent Inpatients. Dusunen Adam: Journal of Psychiatry \& Neurological Sciences, 23(3), 174-184. doi: 10.5350/DAJPN2010230305

Farber, P. D., Khavari, K. A. y Douglass, F. M. (1980). A factor analytic study of reasons for drinking: Empirical validation of positive and negative reinforcement dimensions. Journal of Consulting and Clinical Psychology, 48(6), 780-781. doi: 10.1037//0022-006X.48.6.780

Garimaldi, J., Caneto, F., Vera, B. del V., Tuzinkievich, F. B. y Pilatti, A. (2013, octubre). Patrones de consumo de drogas en adolescentes argentinos y análisis del efecto de la edad del primer consumo de alcohol en el consumo de sustancias. En VIII Congreso Latinoamericano Interdisciplinario del Adolescente (CLIOA) (pp. 35). Córdoba, Argentina.

George, D. y Mallery, M. (2011). SPSS for Windows step by step: A simple guide and reference 18.0 Update (11 ${ }^{\mathrm{a}}$. ed.). Boston, MA: Allyn y Bacon.

Gilson, K. M., Bryant, C., Bei, B., Komiti, A., Jackson, H. y Judd, F. (2013). Validation of the Drinking Motives Questionnaire (DMQ) in older adults. Addictive behaviors, 38(5), 2196-2202. doi: 10.1016/j.addbeh.2013.01.021

Goudriaan, A. E., Grekin, E. R. y Sher, K. J. (2011). Decision making and response inhibition as predictors of heavy alcohol use: A prospective study. Alcoholism: Clinical and Experimental Research, 35(6), 1-8. doi: 10.1111/j.1530-0277.2011.01437.x

Grant, V. V., Stewart, S. H., O'Connor, R. M., Blackwell, E. y Conrod, P. J. (2007). Psychometric evaluation of the fivefactor Modified Drinking Motives Questionnaire - Revised in undergraduates. Addictive Behaviors, 32, 2611-2632. doi: 10.1016/j.addbeh.2007.07.004

Hair, J. F., Black, W. C., Babin, B. J., Anderson, R. E. y Tatham, R. L. (2006). Multivariate Data Analysis. New Jersey: Pearson.

Hauck-Filho, N., Teixeira, M. A. P. y Cooper, M. L. (2012). Confirmatory factor analysis of the Brazilian version of the 
Drinking Motives Questionnaire-Revised (DMQ-R). Addictive behaviors, 37(4), 524-527. doi: 10.1016/j.addbeh.2011.11.023

Hingson, R. W., Edwards, E. M., Heeren, T. y Rosenbloom, D. (2009). Age of drinking onset and injuries, motor vehicle crashes, and physical fights after drinking and when not drinking. Alcoholism: Clinical and Experimental Research, 33(5), 783-790. doi: 10.1111/j.1530-0277.2009.00896.x

Hogan, T. (2004). Pruebas psicológicas: una introducción práctica. México: El Manual Moderno.

Hu, L.-T. y Bentler, P. M. (1999). Cutoff criteria for fit indexes in covariance structure analysis: Conventional criteria versus new alternatives. Structural Equation Modeling, 6, 1-55. doi: 10.1080/10705519909540118

Johnston, L. D., O'Malley, P. M., Bachman, J. G. y Schulenberg, J. E. (2011). Monitoring the future national results on adolescent drug use: Overview of key findings, 2010. Ann Arbor, MI: Institute for Social Research; The University of Michigan.

Kline, R. B. (2011). Principles and practice of structural equation modeling (3rd Ed.). New York: Guilford Press.

Kong, G. y Bergman A. (2010). A motivational model of alcohol misuse in emerging adulthood. Addictive Behaviors, 35, 855860. doi: 10.1016/j.addbeh.2010.06.005

Kuntsche, E., Knibbe, R., Gmel, G. y Engels, R. (2005). Why do young people drink? A review of drinking motives. Clinical Psychology Review, 25(7), 841-861.

Kuntsche, E., Knibbe, R., Gmel, G. y Engels, R. (2006). Who drinks and why? A review of socio-demographic, personality, and contextual issues behind the drinking motives in young people. Addictive behaviors, 31(10), 1844-1857.

Kuntsche, E. y Kuntsche, S. (2009). Development and validation of the Drinking Motive Questionnaire Revised Short Form (DMQ-R SF). Journal of Clinical Child \& Adolescent Psychology, 38(6), 899-908. doi: 10.1080/15374410903258967

Loxton, N. J., Nguyen, D., Casey, L. y Dawe, S. (2008). Reward drive, rash impulsivity and punishment sensitivity in problems gamblers. Personality and Individual Differences, 45, 167-173.

Mason, W. A., Toumbourou, J. W., Herrenkohl, T. I., Hemphill, S. A., Catalano, R. F. y Patton, G. C. (2011). Early age alcohol use and later alcohol problems in adolescents: Individual and peer mediators in a bi-national study. Psychology of addictive behaviors, 25(4), 625. doi: 10.1037/a0023320

Masten, A. S., Faden, V. B., Zucker, R. A. y Spear L. P. (2009). A developmental perspective of underage alcohol use. Alcohol Research and Health, 32, 3-15.

Mazzardis, S., Vieno, A., Kuntsche, E. y Santinello, M. (2010). Italian validation of the drinking motives questionnaire revised short form (DMQ-R SF). Addictive Behaviors, 35(10), 905-908. doi: 10.1016/j.addbeh.2010.05.002

Merrill, J. E. y Read, J. P. (2010). Motivational pathways to unique types of alcohol consequences. Psychology of Addictive Behaviors, 24(4), 705. doi: 10.1037/a0020135

Mezquita, L., Stewart, S. H., Ibáñez, M. I., Ruipérez, M. A., Villa, H., Moya, J. y Ortet, G. (2011). Drinking motives in clinical and general populations. European Addiction Research, 17(5), 250-261. doi: 10.1159/000328510

Morales-García, J. I., Fernández-Gárate, I. H., Tudón Garcés, H., Escobedo de la Peña, J., Zárate-Aguilar, A. y Madrazo-Navarro, M. (2002). Prevalencia de consumo riesgoso y dañino de alcohol en derechohabientes del Instituto Mexicano del Seguro Social. Salud Pública de México, 44, 113- 121.

Mushquash, C. J., Stewart, S. H., Comeau, M. N. y McGrath, P. J. (2008). The structure of drinking motives in first nations adolescents in Nova Scotia. American Indian and Alaska Native Mental Health Research, 15, 33-52.

Neighbors, C., Lee, C. M., Lewis, M. A., Fossos, N. y Larimer, M. E. (2007). Are social norms the best predictor of outcomes among heavy-drinking college students? Journal of Studies on Alcohol and Drugs, 68(4), 556-565.

Németh, Z., Urbán, R., Farkas, J., Kuntsche, E. y Demetrovics, Z. (2012). Hungarian adaptation of the long and the short form of the drinking motives questionnaire (DMQ-R). Magyar Pszichológiai Szemle, 67(4), 673-694.

Németh, Z., Urbán, R., Kuntsche, E., San Pedro, E. M., Nieto, J. G. R., Farkas, J., ... y Demetrovics, Z. (2011). Drinking motives among Spanish and Hungarian young adults: A cross-national study. Alcohol and Alcoholism, 46(3), 261-269. doi: 10.1093/ alcalc/agr019

O'Hare, T. (1997). Measuring excessive alcohol use in college drinking contexts: The Drinking Context Scale. Addictive Behaviors, 22, 469-477.

O'Hare, T. (2001). The Drinking Context Scale. A confirmatory factor analysis. Journal of Substance Abuse Treatment, 20, 129136.

Pilatti, A. y Brussino, S. A. (2009). Construcción y valoración de las propiedades psicométricas del Cuestionario de Contextos de Consumo de Alcohol para Adolescentes (CCCA-A). Revista Argentina de Ciencias del Comportamiento, 1, 13-25.

Pilatti, A., Brussino, S. A. y Godoy, J. C. (2013). Factores que influyen en el consumo de alcohol de adolescentes argentinos: un path análisis prospectivo. Revista de Psicología, 22(1), 2236. doi: 10.5354/0719-0581.2013.27716

Pilatti, A., Caneto, F., Garimaldi, J. A., Vera, B. del V. y Pautassi, R. M. (2014). Contribution of time of drinking onset and family history of alcohol problems in alcohol and drug use behaviors in Argentinean college students. Alcohol and Alcoholism, 49(2),128-37. doi: 10.1093/alcalc/agt176

Pilatti, A., Godoy, J. C., Brussino, S. A. y Pautassi, R. M. (2013a). Patterns of substance use among Argentinean adolescents and analysis of the effect of age at first alcohol use on substance use behaviors. Addictive Behaviors, 38(12), 2847-2850. doi: 10.1016/j.addbeh.2013.08.007

Pilatti, A., Godoy, J. C., Brussino, S. y Pautassi, R. M. (2013b). Underage drinking: Prevalence and risk factors associated to drinking experiences among Argentinean children. Alcohol, 47(4), 323-331. doi: 10.1016/j.alcohol.2013.02.001 
Pilatti, A., Read, J. P., Vera, B. del V., Caneto, F., Garimaldi J. A. y Kahler, C. (2014). The Spanish version of the Brief Young Adult Alcohol Consequences Questionnaire (B-YAACQ): A Rasch Model Analysis. Addictive Behaviors, 39(5), 842-847. doi: 10.1016/j.addbeh.2014.01.026

Read, J. P., Wood, M. D., Kahler, C. W., Maddock, J. E. y Palfai, T. P. (2003). Examining the role of drinking motives in college student alcohol use and problems. Psychology of Addictive Behaviors, 17, 13-23. doi: 10.1037/0893-164X.17.1.13

Reinert, D. F. y Allen, J. P. (2007). The alcohol use disorders identification test: An update of research findings. Alcoholism: Clinical and Experimental Research, 31(2), 185-199.

Satorra, A. (2002). Asymptotic robustness in multiple group linear-latent variable models. Econometric Theory, 18(2), 297312. doi: $10.1017 /$ S0266466602182041

Secretaría de Programación para la Prevención de la Drogadicción y la Lucha contra el Narcotráfico (2011). Algunos datos sobre el consumo de alcohol en Argentina. Sistema de Vigilancia Epidemiológica en Salud Mental y Adicciones. Buenos Aires: Ministerio de Salud.

Shah, R. y Goldstein, S. M. (2006). Use of structural equation modeling in operations management research: Looking back and forward. Journal of Operations Management, 24(2), 148169.

Sher, K. J., Walitzer, K. S., Wood, P. K. y Brent, E. E. (1991). Characteristics of children of alcoholics: Putative risk factors, substance use and abuse, and psychopathology. Journal of Abnormal Psychology, 100(4), 427-448. doi: 10.1037/0021843X.100.4.427
Ståhlbrandt, H., Leifman, A., Johnsson, K. O. y Berglund, M. (2010). Alcohol trajectories over three years in a Swedish residence hall student population. International Journal of Environmental Research and Public Health, 7(4), 1432-1447. doi: 10.3390/ijerph7041432

Urbán, R., Kökönyei, G. y Demetrovics, Z. (2008). Alcohol outcome expectancies and drinking motives mediate the association between sensation seeking and alcohol use among adolescents. Addictive Behaviors, 33, 1344-1352. doi: 10.1016/j. addbeh.2008.06.006

Vera, B. del V., Caneto, F., Garimaldi, J. y Pilatti, A. (2013, agosto). Patrones de consumo de alcohol en mujeres universitarias ingresantes a la facultad de psicología: relación con la edad de inicio. Poster presentado en la XIV Reunión Nacional y III Encuentro Internacional de la Asociación Argentina de Ciencias del Comportamiento, Córdoba, Argentina. Resumen recuperado de http://conferencias.unc.edu.ar/index.php/ AACC/aacc/paper/view/832

Windle, M., Spear, L. P., Fuligni, A. J., Angold, A., Brown, J. D., Pine, D., ... Dahl, R. E. (2009). Transitions into underage and problem drinking. Alcohol Research \& Health, 32, 30-40. doi: 10.1542/peds.2007-2243C

World Health Organization (2010). Global Status Report on Noncommunicable Diseases. Ginebra: World Health Organization.

Zogmaister, C. y Castelli, L. (2011). Perché i giovani bevono? Versione italiana del DMQ-R (Cooper, 1994), strumento per l'indagine della motivazione al consumo di alcoolici. Psicologia della Salute, 1, 101-118. doi: 10.3280/pds2011-001008 